Optical studies of molecular beam epitaxy grown GaAsSbN / GaAs single quantum well structures

Kalyan Nunna, S. Iyer, L. Wu, S. Bharatan, Jia Li, K. K. Bajaj, X. Wei, and R. T. Senger

Citation: Journal of Vacuum Science \& Technology B: Microelectronics and Nanometer Structures Processing, Measurement, and Phenomena 25, 1113 (2007); doi: 10.1116/1.2720860

View online: http://dx.doi.org/10.1116/1.2720860

View Table of Contents: http://avs.scitation.org/toc/jvn/25/3

Published by the American Institute of Physics 


\title{
Optical studies of molecular beam epitaxy grown GaAsSbN/GaAs single quantum well structures
}

\author{
Kalyan Nunna, ${ }^{a)}$ S. Iyer, L. Wu, S. Bharatan, and Jia Li \\ North Carolina A\&T State University, Greensboro, North Carolina 27411 \\ K. K. Bajaj \\ Department of Physics, Emory University, Atlanta, Georgia 30322 \\ X. Wei \\ NHMFL, Florida State University, Tallahassee, Florida 32310 \\ R. T. Senger \\ Physics Department, Bilkent University, Ankara 06800, Turkey
}

(Received 6 November 2006; accepted 5 March 2007; published 31 May 2007)

\begin{abstract}
In this work, the authors present a systematic study on the variation of the structural and the optical properties of GaAsSbN/GaAs single quantum wells (SQWs) as a function of nitrogen concentration. These SQW layers were grown by the solid source molecular beam epitaxial technique. A maximum reduction of $328 \mathrm{meV}$ in the photoluminescence (PL) peak energy of GaAsSbN was observed with respect to the reference GaAsSb QW. $8 \mathrm{~K}$ and RT PL peak energies of $0.774 \mathrm{eV}$ (FWHM of $\sim 25 \mathrm{meV}$ ) and $0.729 \mathrm{eV}$ (FWHM of $\sim 67 \mathrm{meV}$ ) (FWHM denotes full width at half maximum) corresponding to the emission wavelengths of 1.6 and $1.7 \mu \mathrm{m}$, respectively, have been achieved for a GaAsSbN SQW of $\mathrm{N} \sim 1.4 \%$. The pronounced $S$-curve behavior of the PL spectra at low temperatures is a signature of exciton localization, which is found to decrease from 16 to $9 \mathrm{meV}$ with increasing $\mathrm{N}$ concentration of $0.9 \%-2.5 \%$. The diamagnetic shift of $13 \mathrm{meV}$ observed in the magnetophotoluminescence spectra of the nitride sample with $\mathrm{N} \sim 1.4 \%$ is smaller in comparison to the value of $28 \mathrm{meV}$ in the non-nitride sample, indicative of an enhancement in the electron effective mass in the nitride QWs. Electron effective mass of $0.065 m_{o}$ has been estimated for a SQW with $\mathrm{N} \sim 1.4 \%$ using the band anticrossing model. (C) 2007 American Vacuum Society. [DOI: 10.1116/1.2720860]
\end{abstract}

\section{INTRODUCTION}

InGaAsN/GaAs, $\mathrm{GaAsSbN} / \mathrm{GaAs}$, and InGaAs( $\mathrm{Sb}) \mathrm{N} / \mathrm{GaAs}$ dilute nitride alloy systems lattice matched to GaAs are of potential interest in optical communications in the $1.55 \mu \mathrm{m}$ emission wavelength region. InGaAsN/GaAs has been the most extensively studied system; however, the operating wavelength of $1.55 \mu \mathrm{m}$ necessitates $\mathrm{N}$ and In concentrations exceeding $2 \%$ and $35 \%$, respectively, leading to considerable degradation of the structural and optical properties. ${ }^{1-4}$ More recently InGaAsN(Sb)/GaAs system has been demonstrated to be successful in this wavelength range ${ }^{2,3,5}$ but five components make the system more complex. The work on GaAsSbN/GaAs system has been somewhat limited. ${ }^{6-11}$ Our earlier work on the GaAsSbN quantum wells ${ }^{6}$ (QWs) shows that good quality structures with emission at $1.55 \mu \mathrm{m}$ can be reached for a $\mathrm{N}$ concentration of $\sim 1.4 \%$.

In this work, we present a detailed and systematic study of the effect of $\mathrm{N}$ incorporation on the structural, low temperature photoluminescence characteristics and the calculated conduction band electron effective mass values. The temperature dependence of PL characteristics in the low temperature regime is dominated by localized excitons caused

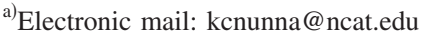

by the potential fluctuations introduced by $\mathrm{N}$ incorporation. The exciton localization was found to become weaker with increasing $\mathrm{N}$ concentration. The magnetophotoluminescence data indicate enhanced conduction band (CB) electron effective mass in nitride QWs in comparison to the non-nitride QWs. The computation of the enhanced effective mass value using band anticrossing (BAC) model is also presented.

\section{EXPERIMENTAL DETAILS}

The GaAsSbN/GaAs single QW (SQW) structures were grown using the solid source molecular beam epitaxial technique with $\mathrm{N}$ plasma source. The GaAsSbN QW layers were sandwiched between GaAs layers followed by GaAlAs to improve carrier confinement in the QWs. The growth temperatures of the QWs and GaAlAs barriers were 470 and $580{ }^{\circ} \mathrm{C}$, respectively, and these samples were exposed to $\mathrm{Sb}$ and As flux prior to QW growth. All the samples were subjected to in situ annealing in As ambient at $650{ }^{\circ} \mathrm{C}$ to improve the luminescence. ${ }^{6}$ High resolution $\mathrm{X}$-ray diffraction (HRXRD) was performed with a Bede Scientific Metrix-F automated diffractometer, equipped with a microsource X-ray generator. The motorized detector slit was set to $0.5 \mathrm{~mm}$ wide, giving a $2 \theta$ angular resolution of $150 \mathrm{arcsec}$. The compositions of $\mathrm{Sb}$ and $\mathrm{N}$ were determined using simulation of the HRXRD spectra and secondary ion mass spectroscopy data. The $\mathrm{Sb}$ composition in all the samples was in the range 


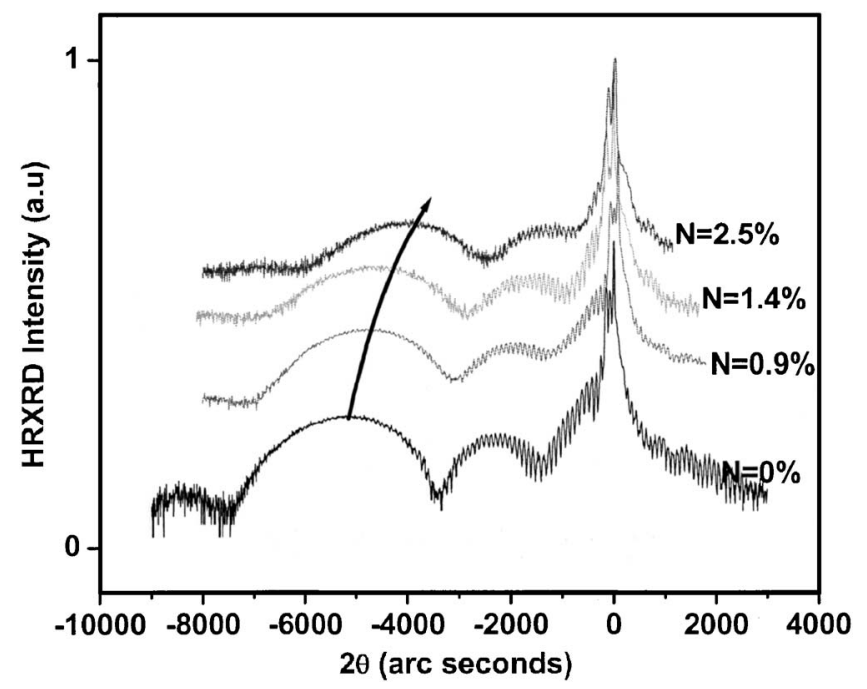

FIG. 1. (004) HRXRD $\theta-2 \theta$ scan spectra for GaAsSbN SQWs with increasing $\mathrm{N}$ incorporation.

of $28 \%-30 \%$. The details of the PL measurements are given in Ref. 6. Low temperature (4 K) magnetophotoluminescence measurements were carried out with the magnetic field varying from 0 to $32 \mathrm{~T}$ and were directed normal to the sample. The details of these are given in Ref. 7.

\section{RESULTS}

Figure 1 illustrates the HRXRD spectra of GaAsSbN SQW with GaAlAs barriers grown for various $\mathrm{N}$ concentrations. With the introduction of small amount of $\mathrm{N}(0.9 \%-$ $2.5 \%$ ) the quaternary GaAsSbN layer peak shifts to the right of the compressively strained reference GaAsSb QW peak grown under similar conditions. Pendullosung fringes are observed in all the samples. $\mathrm{N}$ composition in the SQWs increases almost linearly but only a marginal increase in the $\mathrm{Sb}$ composition with increasing $\mathrm{N}$ flux is observed.

Figure 2 displays the low temperature $(10 \mathrm{~K})$ and room temperature PL spectra of the GaAsSbN SQW structure for $\mathrm{N} \sim 1.4 \%$ grown at $470{ }^{\circ} \mathrm{C}$, along with those of the reference GaAsSb SQW for comparison. The $10 \mathrm{~K}$ PL spectral position of the quaternary SQW shifted to lower energy by $\sim 328 \mathrm{meV}$ exhibited a somewhat higher PL linewidth of $25 \mathrm{meV}$ and a lower intensity as compared to the non-nitride QW. The PL peak wavelengths corresponding to a $10 \mathrm{~K}$ emission of $1.6 \mu \mathrm{m}$ (FWHM of $\sim 25 \mathrm{meV}$ ) and a RT emission of $1.7 \mu \mathrm{m}$ (FWHM of $\sim 67 \mathrm{meV}$ ) (FWHM denotes full width at half maximum) have been achieved. The PL peak energy decreases rapidly up to $\mathrm{N} \sim 1.4 \%$ but thereafter the reduction in PL peak energy seizes, as shown in Fig. 3.

Figure 4 shows the temperature dependence of the PL spectra of the SQWs for N concentrations of $0 \%, 0.9 \%$, $1.4 \%$, and $2.5 \%$. The low temperature PL peak energy exhibits redshift and blueshift with increasing temperature up to $\sim 100 \mathrm{~K}$ and thereafter decreases monotonically with temperature. This characteristic $S$-curve behavior becomes less pronounced with the increasing $\mathrm{N}$ concentration (Fig. 4).

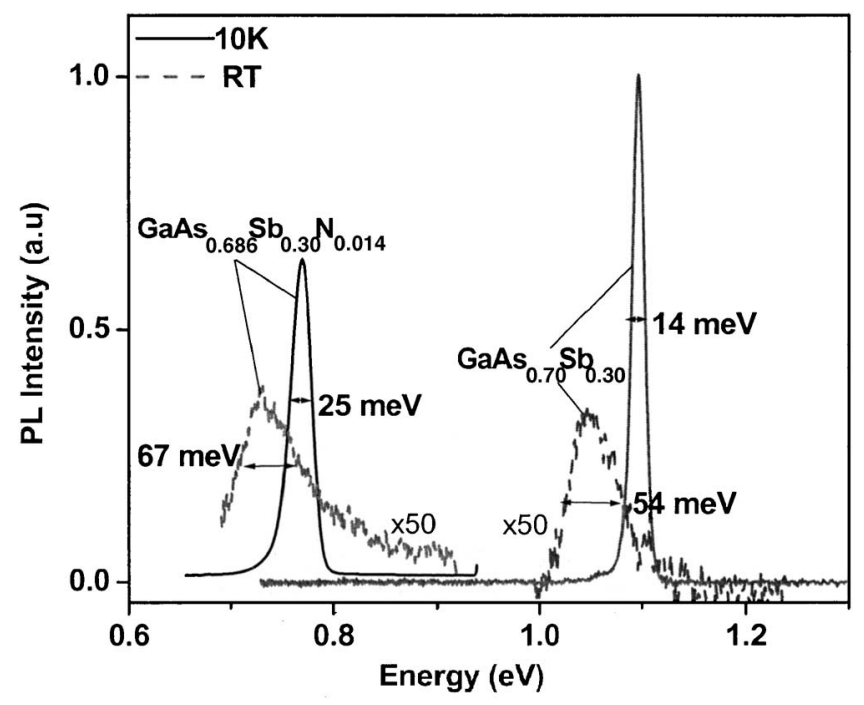

FIG. 2. $10 \mathrm{~K}$ and RT PL spectra of GaAsSb and in situ annealed GaAsSbN $(\mathrm{N} \sim 1.4 \%)$ SQWs.

The temperature dependence of PL peak energy of the SQWs was fitted using the well-known Varshni empirical relation, ${ }^{8}$

$$
E_{g}(T)=E_{g}(0)-\frac{\alpha T^{2}}{\beta+T},
$$

where $T$ is the absolute temperature, $E_{g}(0)$ is the band gap at $0 \mathrm{~K}$, and $\alpha$ and $\beta$ are the fitting parameters. The values of these parameters for all the samples are tabulated in Table I. This table also lists the values of the parameters $T_{\text {trans }}, E_{\text {loc }}^{\max }$, and $T_{\text {deloc }}$ which are defined as the onset of the transition temperature regime where localized excitons begin transition to higher energy localized states, the maximum localization energy measured as the largest energetic difference between the experimental PL peak energy and value of the energy obtained using Varshni relation, and the temperature at which

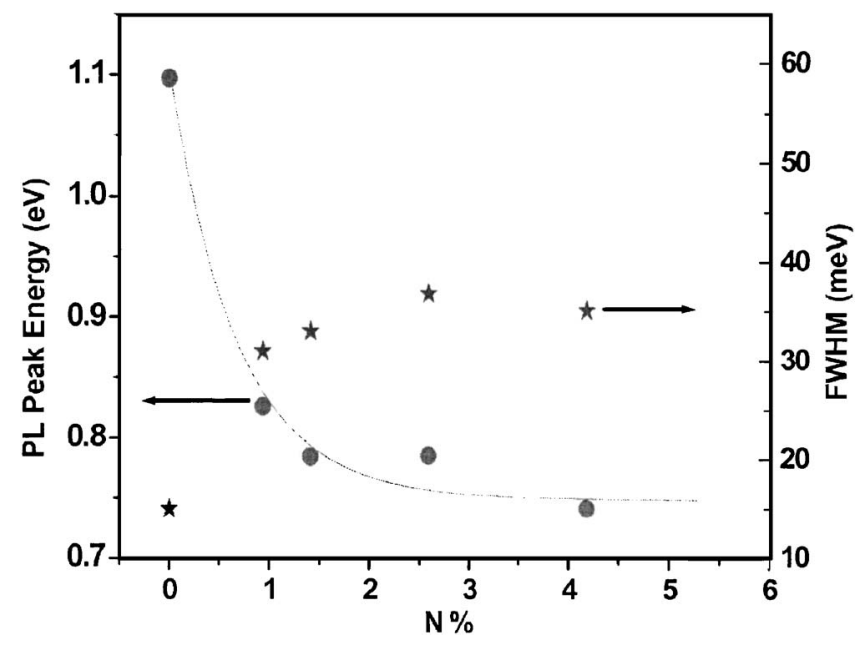

FIG. 3. $10 \mathrm{~K}$ PL peak energy shifts and FWHM variations for different N concentrations. The solid line represents the trend line of variation of the PL peak energy with $\mathrm{N}$ concentration. 


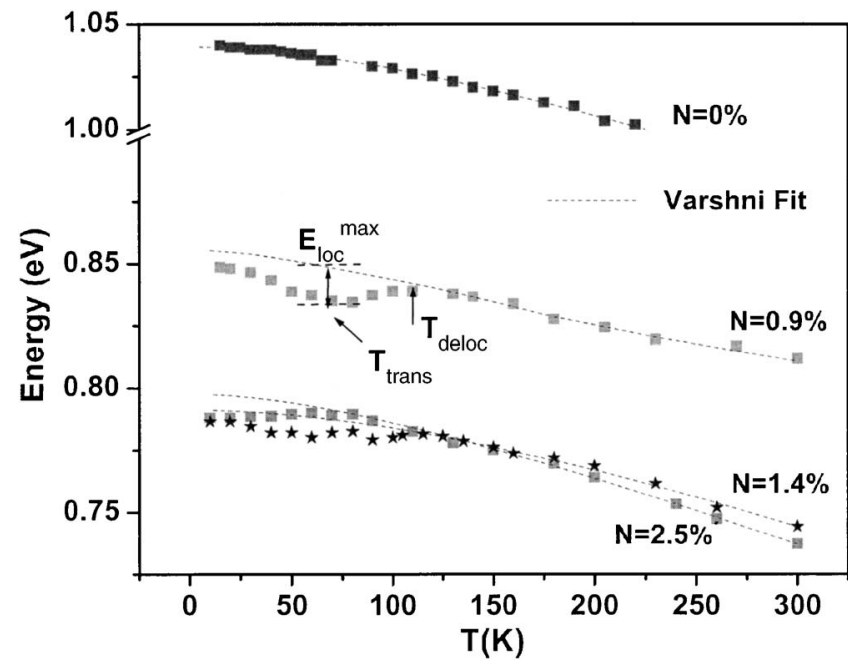

FIG. 4. Temperature dependence of QWs for different $\mathrm{N}$ concentrations illustrating the $S$-curve behavior of the PL peak position and the Varshni fitting [Eq. (1)].

delocalization of the carriers is complete, respectively. The values of these parameters are found to decrease with increase in $\mathrm{N}$ concentration.

Figure 5 depicts a magneto-PL spectrum of GaAsSbN SQW for $\mathrm{N} \sim 1.4 \%$. A blueshift of $13 \mathrm{meV}$ (28 meV shift in $\mathrm{GaAsSb} \mathrm{QW}$ ) and broadening of the spectra are observed in the presence of magnetic field $(32 \mathrm{~T})$ as expected. ${ }^{7,12,13}$ The variation of diamagnetic shift, $\delta$ defined as $E_{g}(B)-E_{g}(B$ $=0$ ), as a function of the applied magnetic field $(B)$ for $\mathrm{N}$ $\sim 1.4 \%$ is shown in Fig. 6 . The $\delta$ observed in GaAsSb SQW vary linearly with $B$ while the variation in GaAsSbN SQW is sublinear and is significantly less than that observed in $\mathrm{GaAsSb}$ reference QW. The effective mass of the nitride QW has been estimated using the BAC model ${ }^{14}$ as follows. The standard equation obtained from the definition of density of states to calculate the effective mass is

$$
m_{\mathrm{eff}}=m^{*}\left\{1+\left[\frac{V_{N M}}{E_{N}-E_{M}(k)}\right]^{2}\right\},
$$

where the resonant energy level $E_{N}$, introduced by $\mathrm{N}$ is assumed to be around $1.65 \mathrm{eV}$ above the valence band edge as in InGaAsN and GaAsN systems, ${ }^{14,15}$ and $E_{M}(k)$ is the interacting CB energy level of the host semiconductor GaAsSb. In the above equation, the value of $V_{N M}$ is computed using the low temperature PL peak energy in the energy dispersion relation (see Refs. 14 and 15).

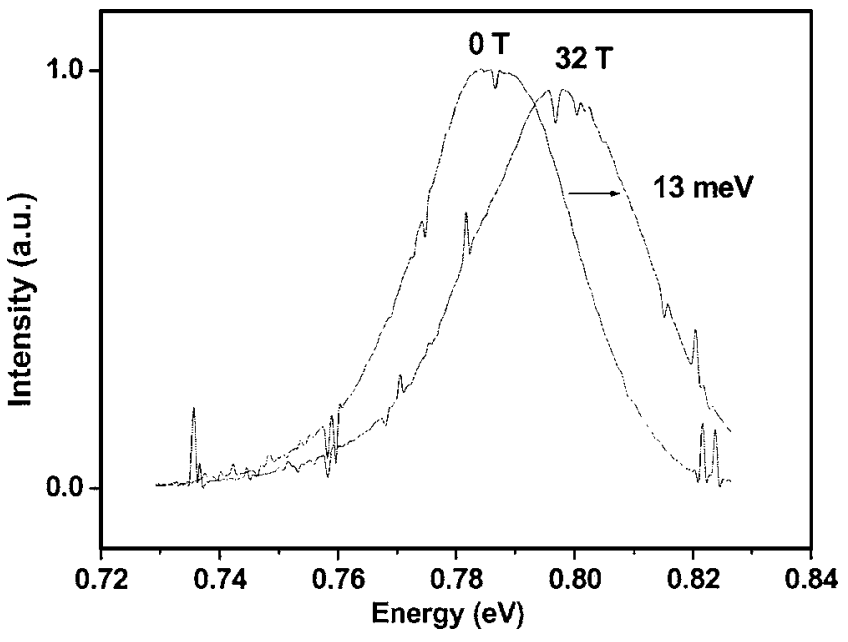

FIG. 5. Magento-PL spectra of GaAsSbN $(\mathrm{N} \sim 1.4 \%) \mathrm{SQW}$ at $4 \mathrm{~K}$ at 0 and $32 \mathrm{~T}$.

Using $m^{*}=0.050 m_{0}$, for the electron effective mass in the host GaAsSb at low energies and low values of the wave vector $k, m_{\text {eff }}$ for the GaAsSbN SQW is computed to be $\sim 0.065 m_{o}$ for the $\mathrm{N}$ concentration of $\sim 1.4 \%$.

\section{DISCUSSION}

The presence of Pendullosung fringes in the x-ray rocking curves attest to the excellent quality of the grown layers and abrupt interfaces (Fig. 1). The ternary GaAsSb QWs are compressively strained and with increasing $\mathrm{N}$ concentration, the strain in the QW layer becomes more tensile. Sb incorporation is found to be relatively independent of $\mathrm{N}$ composition in our QWs.

The PL peak energy decreases at a faster rate for $\mathrm{N}$ concentrations up to $1.4 \%$, as shown in Fig. 3. A reduction of $\sim 328 \mathrm{meV}$ in energy has been observed in the GaAsSbN $\mathrm{SQW}$ for $\mathrm{N} \sim 1.4 \%$ and $\mathrm{Sb} \sim 30 \%$ with reference to $\mathrm{GaAsSb}$ $\mathrm{SQW}$, which is much larger than the reported values ${ }^{7,9-11}$ in this material system.

$S$-curve behavior observed at low temperatures in the temperature dependence of the PL peak energy position of the GaAsSbN SQWs, as shown in Fig. 4, is commonly attributed to the localized behavior of excitons due to the potential fluctuations arising from the compositional variation and/or $\mathrm{N}$ related defects. The observed trend in the localization energy and transition and delocalization temperatures with $\mathrm{N}$ concentration can be explained qualitatively as follows. The

TABLE I. Varshni's parameters for the temperature dependence of the PL peak energy for different $\mathrm{N}$ concentrations.

\begin{tabular}{lcccccc}
\hline \hline $\mathrm{N} \%$ & $E_{10 \mathrm{~K}}$ & $\begin{array}{c}\alpha \\
(\mathrm{meV} / \mathrm{K})\end{array}$ & $\begin{array}{c}\beta \\
(\mathrm{K})\end{array}$ & $\begin{array}{c}E_{\mathrm{loc}}^{\max } \\
(\mathrm{meV})\end{array}$ & $\begin{array}{c}T_{\text {trans }} \\
(\mathrm{K})\end{array}$ & $\begin{array}{c}T_{\text {deloc }} \\
(\mathrm{K})\end{array}$ \\
\hline 0 & 1.039 & 0.36 & 245 & $\ldots$ & $\ldots$ & $\ldots$ \\
0.9 & 0.823 & 0.37 & 273 & 16 & 75 & 100 \\
1.4 & 0.786 & 0.36 & 325 & 8 & 60 & 90 \\
2.5 & 0.788 & 0.33 & 204 & 9 & 10 & 80 \\
\hline \hline
\end{tabular}




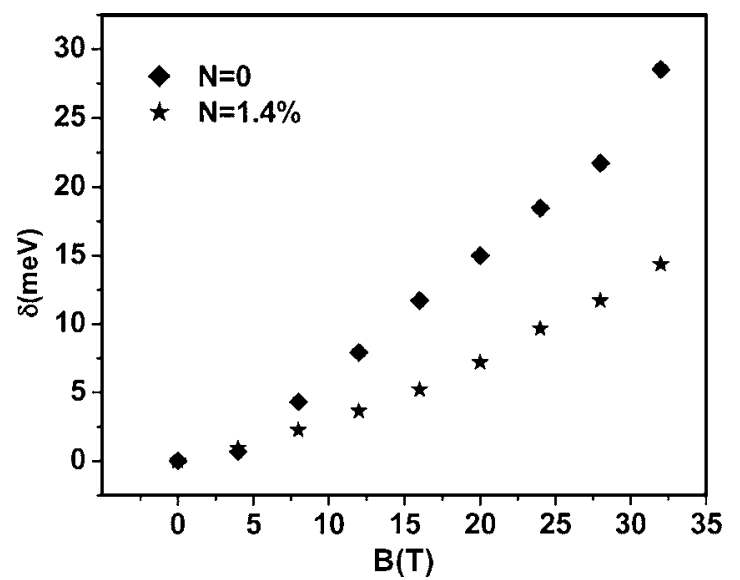

FIG. 6. Variation of the diamagnetic shift as a function of the magnetic field for the ternary and the quaternary SQWs.

nitride QW at low $\mathrm{N}$ concentration of $0.9 \%$ exhibits the highest localization energy of about $16 \mathrm{meV}$. The excitons require elevated temperatures as high as $\sim 80-100 \mathrm{~K}$ to detrap from the potential wells. As the $\mathrm{N}$ concentration increases, these deeper potentials appear to become shallower as evidenced by the less pronounced $S$ curve. This unexpected trend could be attributed to any or combination of the following effects. These are decreased alloy fluctuations, increased confinement of carriers screening these potential modulations,${ }^{16}$ and effective annihilation of $\mathrm{N}$ related defect centers.

The PL transition energy in the presence of the magnetic field exhibits a smaller shift in the nitride QWs in comparison to the non-nitride reference sample, implying an enhancement in the electron effective mass value. The electron effective mass calculated using the BAC model for the quaternary QW is $0.065 m_{o}$ lower than those computed by Senger et $\mathrm{al}^{7}\left(0.09 \mathrm{~m}_{o}\right)$ in a GaAsSbN QW for similar N concentration. This is consistent with the larger $\delta$ observed for a given magnetic field in both the non-nitride and nitride QWs as our values are almost double than those reported by Senger et $a l^{7}$ This suggests that the exciton wave function in our samples is weakly localized. It is to be noted that these are preliminary data and further work is being carried out to determine the conduction electron effective mass more accurately.

\section{CONCLUSIONS}

We have achieved $8 \mathrm{~K}$ and RT PL emission at $1.6 \mu \mathrm{m}$ (FWHM of $\sim 25 \mathrm{meV}$ ) and $1.7 \mu \mathrm{m}$ (FWHM of $\sim 67 \mathrm{meV}$ ) in GaAsSbN SQW for $\mathrm{N} \sim 1.4 \%$. Temperature dependence of the PL spectra exhibited exciton localization at temperatures below $\sim 100 \mathrm{~K}$. PL peak energy reduction of $328 \mathrm{meV}$ from the reference GaAsSb has been observed. The changes in the PL characteristics are significant up to $\mathrm{N} \sim 1.4 \%$ and thereafter saturates. The exciton localization energy and delocalization temperature were found to decrease with increase in $\mathrm{N}$ concentration. Electron effective mass is enhanced in nitride QWs in comparison to the non-nitride QWs as expected.

\section{ACKNOWLEDGMENTS}

This work is supported by Army Research Office (Grant No. W911NF-04-1-0025). High resolution x-ray diffraction scans were done by Dr. Kevin Matney at Bede Scientific Inc., Englewood, CO. Magneto-PL measurements were done at National High Magnetic Field Laboratory, Tallahassee.

${ }^{1}$ H. Saito, T. Makimoto, and N. Kobayashi, J. Cryst. Growth 195, 416 (1998).

${ }^{2}$ Kerstin Volz, Vincent Gambin, Wonill Ha, Mark A. Wistey, Homan Yuen, Seth Bank, and James S. Harris, J. Cryst. Growth 251, 360 (2003).

${ }^{3}$ L. H. Li, V. Sallet, G. Patriarche, L. Largeau, S. Bouchoule, L. Travers, and J. C. Harmand, Appl. Phys. Lett. 83, 1298 (2003).

${ }^{4}$ M.-A. Pinault and E. Tournie, Solid-State Electron. 47, 477 (2003).

${ }^{5}$ James S. Harris, Jr., J. Cryst. Growth 278, 3 (2005).

${ }^{6}$ L. Wu, S. Iyer, K. Nunna, J. Li, S. Bharatan, W. Collis, and K. Matney, J. Cryst. Growth 279, 293 (2005).

${ }^{7}$ R. T. Senger et al., Appl. Phys. Lett. 83, 5425 (2003).

${ }^{8}$ J. Li, S. Iyer, S. Bharatan, L. Wu, K. Nunna, W. Collis, K. Bajaj, and K. Matney, J. Appl. Phys. 98, 013703 (2005).

${ }^{9}$ J. C. Harmand et al., J. Cryst. Growth 553, 227 (2001).

${ }^{10} \mathrm{G}$. Ungaro, G. Le Roux, R. Teissier, and J. C. Harmand, Electron. Lett. 35, 1246 (1999).

${ }^{11}$ J. C. Harmand et al., Semicond. Sci. Technol. 17, 778 (2002).

${ }^{12}$ J. Zeman, G. Martinez, K. K. Bajaj, I. Krivorotov, and K. Uchida, Appl. Phys. Lett. 77, 4335 (2000).

${ }^{13}$ R. T. Senger et al., Appl. Phys. Lett. 83, 2614 (2003).

${ }^{14}$ C. Skierbiszewski et al., Appl. Phys. Lett. 76, 2409 (2000).

${ }^{15}$ J. Wu, W. Shan, W. Walukiewicz, K. M. Yu, J. W. Ager III, E. E. Haller, H. P. Xin, and C. W. Tu, Phys. Rev. B 64, 085320 (2001).

${ }^{16}$ H. Dumont, L. Auvray, Y. Montiel, F. Saidi, F. Hassen, and H. Marref, Opt. Mater. (Amsterdam, Neth.) 24, 303 (2003). 